\title{
Digital design and fabrication of simulation model for measuring orthodontic force
}

\author{
Yun-Feng Liu ${ }^{\mathrm{a},{ }^{*}}$, Peng-Yuan Zhang ${ }^{\mathrm{a}}$, Qiao-Fang Zhang ${ }^{\mathrm{a}}$, Jian-Xing Zhang ${ }^{\mathrm{b}}$ and Jie Chen ${ }^{\mathrm{c}}$ \\ ${ }^{a}$ Key Laboratory of E\&M (Zhejiang University of Technology), Ministry of Education \& Zhejiang \\ Province, Hangzhou 310014, China \\ ${ }^{b}$ Department of Stomatology, Zhejiang People's Hospital, Hangzhou 310014, China \\ ${ }^{c}$ Department of Mechanical Engineering and Department of Orthodontics and Oral Facial Genetics, \\ Indiana University Purdue University, Indianapolis, IN, 46202, USA
}

\begin{abstract}
Three dimensional (3D) forces are the key factors for determining movement of teeth during orthodontic treatment. Designing precise forces and torques on tooth before treatment can result accurate tooth movements, but it is too difficult to realize. In orthodontic biomechanical systems, the periodontal tissues, including bones, teeth, and periodontal ligaments (PDL), are affected by braces, and measuring the forces applied on the teeth by braces should be based on a simulated model composed of these three types of tissues. This study explores the design and fabrication of a simulated oral model for 3D orthodontic force measurements. Based on medical image processing, tissue reconstruction, 3D printing, and PDL simulation and testing, a model for measuring force was designed and fabricated, which can potentially be used for force prediction, design of treatment plans, and precise clinical operation. The experiment illustrated that bi-component silicones with 2:8 ratios had similar mechanical properties to PDL, and with a positioning guide, the teeth were assembled in the mandible sockets accurately, and so a customized oral model for 3D orthodontic force measurement was created.
\end{abstract}

Keywords: Orthodontic force measurement, oral model, reconstruction, 3D printing

\section{Introduction}

The movement of teeth during orthodontic treatment should be controlled in all three planes of space, including the crown and root position. To move a tooth into the ideal position according to a designed direction and steps using a 3D force applied to the teeth by braces, force and movement should be accurately known. Without a clear understanding of forces and movements of the teeth, undesirable tooth movements during orthodontic treatment may take place, which could result in added visits and adjustments, failure of the treatment, or complications of root resorption [1].

The periodontal tissues, including tooth roots, PDL, and bones, are affected by orthodontic force, and regenerate and rebuild under a stress environment [2]; understanding the biomechanical system of tooth movement can provide the ability to accurately determine the level of stress in various areas of the periodontal tissues [3] to create the relationship between the applied force on a tooth and the

\footnotetext{
${ }^{*}$ Corresponding author: Yun-Feng Liu, Key Laboratory of E\&M (Zhejiang University of Technology), Ministry of Education \& Zhejiang Province, Hangzhou 310014, China. Tel.: +86 159 67144532; Fax: +86 571 88320339; E-mail: liuyf76@126.com.
}

0959-2989/14/\$27.50 @ 2014 - IOS Press and the authors. 
tooth's response. In recent years, most research on orthodontic biomechanics has been restricted to 3D computer modeling and virtual simulation by finite element method (FEM) [4,5], although some experimental measurements and analyses of orthodontic force systems have been proposed and performed $[1,6]$. To measure the force on a tooth, intraoral measurements on braces is real-time and more accurate, but it is very difficult to realize in the limited space of the oral cavity with the large size of the force sensor [7]. Because of these difficulties, extra-oral measurements on simulated oral models are a more feasible method with the current technical tools.

To study the orthodontic force system in three dimensions from the extra oral measurements on simulated models, a system is required that includes force sensors capable of measuring $3 \mathrm{D}$ forces and movements, a simulated oral model composed of periodontal tissues, a frame for integrating the sensors and oral model, and a computer system of software and hardware for data collection, display and recording. The sensors, therefore, must be small, of a size that can be used on neighboring teeth. Multi-axis force transducers called Nano17 (ATI, USA), which is the smallest sensor for 3D force measurement in the world, were used for 3D orthodontic force measurements [1].

\section{Architecture of orthodontic force measurement}

The conceptual structure of the testing device was designed as shown in Figure 1. To measure the 3D forces imposed on the teeth by the braces arch-wires, two Nano17s were used, which were mounted on a track and able to slide along the track to measure different teeth after being locked in position by screws. The transducers were also connected to the two teeth being measured by contact bars. The oral model was fixed to the table by two screws. All the parts were mounted on a steel base. The forces tested by the transducers were collected by a data collector connected to a computer, and then recorded and displayed by self-developed software. According to the parameters of the Nano17, as shown in Table 1 [8], the distance between the contact point and the transducer's gravity center should be limited to $35 \mathrm{~mm}$.

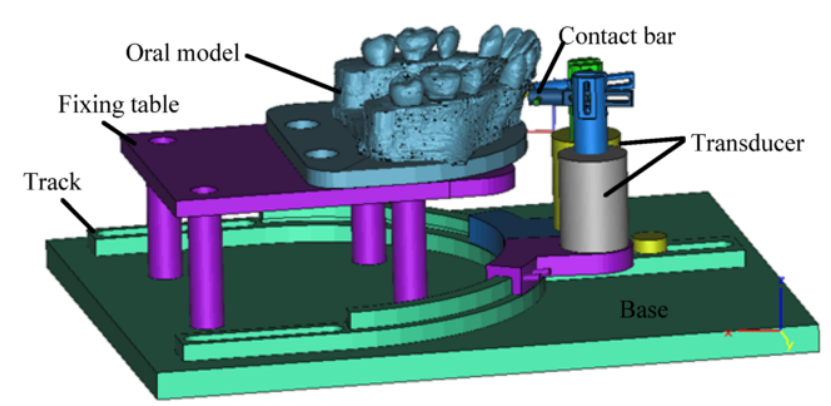

Fig. 1. Orthodontic force measurement system.

Table 1

Parameters of Nano17

\begin{tabular}{|l|l|l|l|}
\hline Items & Value & Sensing ranges & Value \\
\hline Max Fxy $( \pm N)$ & 50 & Fx, Fy $( \pm N)$ & 12 \\
\hline Max Txy $( \pm N-m)$ & 0.5 & Fz $( \pm N)$ & 17 \\
\hline Weight $(\mathrm{Kg})$ & 0.01 & $\mathrm{Tx}, \mathrm{Ty}( \pm \mathrm{Nmm})$ & 120 \\
\hline Diameter $\times$ Height $(\mathrm{mm})$ & $17 \times 14.5$ & $\mathrm{Tz}( \pm \mathrm{Nmm})$ & 120 \\
\hline
\end{tabular}


In this measurement system, only two transducers were arranged, which was based on the consideration of their high cost and the limited space between two teeth for transducer mounting. Only two teeth could be tested at one time, but it was adequate because generally only several teeth at typical positions are focused on during biomechanical analysis and the three forces along the three axes and three torques rotated three axes of a tooth could be acquired concurrently. In this system, the oral model was the key to acquiring personalized values of force.

To measure the force on a tooth imposed by the braces arch-wire, the oral model with brackets bonded on the teeth was fixed on the table first, and then the contact bar of the transducer was linked with the tooth-socket element, which was a separate part composed of the tooth and socket, and semilinked to the entire model by several small cylinders. After the arch-wire was mounted into slots of brackets, the tooth element was fully separated from the model by cutting the linking cylinder, and the force on the tooth loaded by the arch-wire could be sensed by the linked transducer. The design of the tooth element will be discussed in detail in the next section.

\section{Digital design of oral model}

In order to measure the orthodontic force, an oral model including teeth, PDL, and alveolar bones was constructed, and each tooth, as well as each PDL, was created as separate parts, and then assembled with the bone. To describe the design solution conveniently, a typical orthodontic case of canine retraction for upper teeth was selected as an example, and the model construction was based on the original data acquired from a CT scan of a patient.

The image data belonged to a female patient 20 years old. She visited the dentist's office for irregular dentition. Based on the diagnosis, a treatment solution was designed and adopted for the upper teeth with extractions of two first premolars on both sides and then retraction of the canines. This measurement model for orthodontic force utilized the status of the canine retraction with braces after the two premolars were extracted.

\subsection{CT images acquisition}

For data acquisition, both CBCT (cone-beam computerized tomography) for dentistry and general spiral CT are suitable, but for spiral CT, the interval between two slices should be smaller than $1 \mathrm{~mm}$ to guarantee a precisely reconstructed model. In this case for the orthodontic force model, the scan was carried out on a CBCT scanner (KaVo Dental GmbH, Biberach, Germany). The images of the maxillofacial region (158 images) were recorded on a disc in a DICOM format (Digital Imaging and Communications in Medicine) file. During scanning, the patient's mouth was kept open with a cotton roll to prevent the dentitions from touching during scanning, so the maxilla and each tooth could be separately reconstructed easily.

\subsection{Reconstruction and redesign of mandible, teeth, and PDLs}

The images were imported and processed with medical software of a popular commercial platform, Mimics (version 11.04, Materialise NV). With this tool, the images were processed for the construction of a 3D model of the hard tissues in three steps: threshold of the Hounsfield value setting, growing region, and 3D model calculation. With the minimum threshold at 317 , the teeth were separated and reconstructed, as shown in Figure 2. 

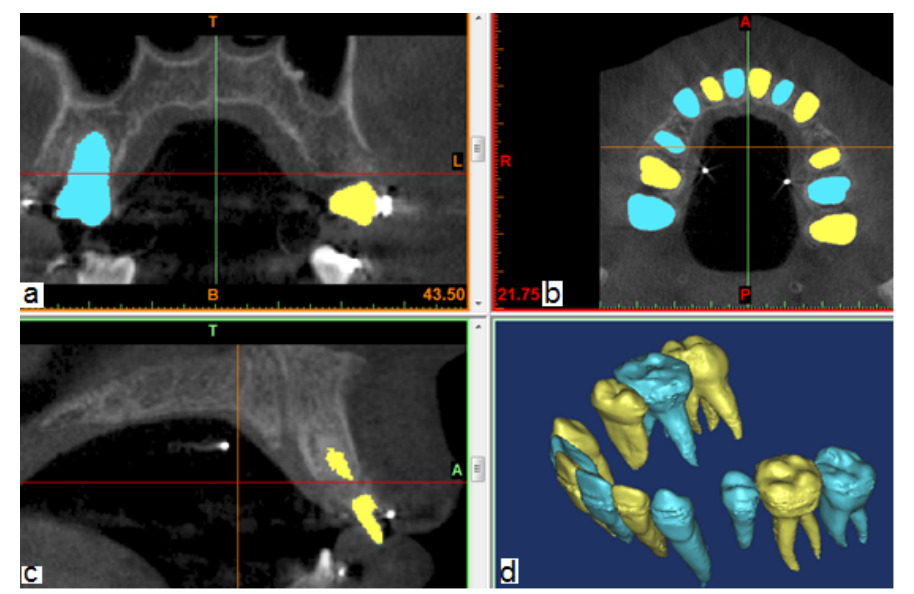

Fig. 2. Tooth reconstruction (a) coronal plane (b) Axial plane (c) sagittal plane (d) 3D view.

The models of the teeth were then exported by STL (Stereography) files, and then processed by Geomagic, a tool for triangular mesh editing. First, the bracket was removed on each tooth, and the tooth surface was filled and smoothed. Then, in order to create space for the PDL, the surface of the tooth model was offset $0.25 \mathrm{~mm}$ outward, which would be used to be subtracted from the maxilla to create a hole with PDL thickness.

Then, with a threshold of 100-317, the maxilla was separated and reconstructed, as shown in Figure 3(a). On the fabricated models, the molars with three roots could not be inserted into holes on maxilla because the curved roots and angles between two roots existed. So, the four molars were designed to link with the maxilla bone together, and the holes of other tooth roots were filled by mesh editing. A basic plate with two holes for fixing was also designed, which was parallel to the occlusal plane, as shown in Figure 3(b). The models of separated teeth with $2.5 \mathrm{~mm}$ surface offset were subtracted from the maxilla bone to create holes for PDL space, and the bone under the lateral incisors and canines for testing were separated from the maxilla to create a tooth-socket element, which was semi-linked by several small cylinders with bordering bone, as shown in Figures 3(c) and 3(d).

\subsection{Positioning guide for teeth}

When inserting teeth into their sockets, the correct position of each tooth was found, which ensured the distance between the root surface and socket wall stayed uniform at $0.25 \mathrm{~mm}$. A guide for inserting was designed for solving this problem, as shown in Figure 4. The position of the guide was determined by the four molars and a positioning cylinder, as marked by ellipses in Figure 4. For each tooth, during the teeth was assembled, and positioned by surface contact between the crown and corresponding hole on the guide, it was fully positioned in the 3D space. To design this guide in Geomagic, a block of $4 \mathrm{~mm}$ thickness was designed. Then, the block was placed in the appropriate position by making it parallel to the basic plate, and the tips of the teeth surpassed the upper plane by approximately $10 \mathrm{~mm}$. After the teeth were subtracted from the block, the redundant parts removed, and the positioning cylinder was designed, the final guide was acquired, as shown in Figure 4(b). 

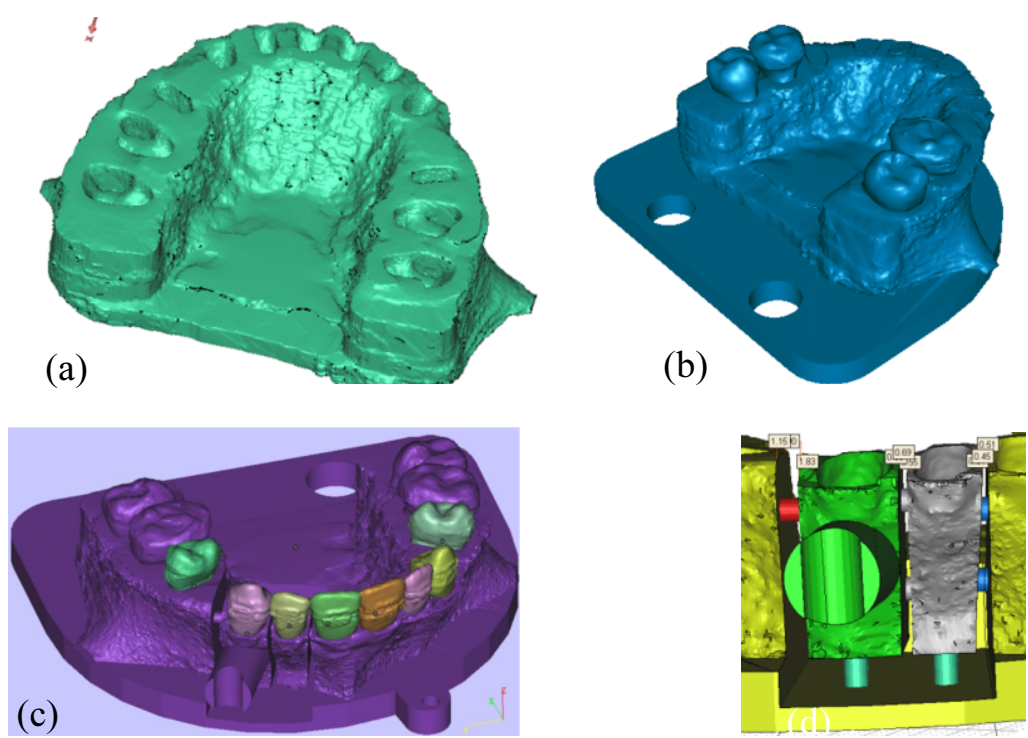

Fig. 3. Oral model reconstruction and design (a) original maxilla (b) maxilla with a basic plate, four molars merged and sockets filled (c) final design model with teeth, tooth-socket complex for testing (d) tooth-socket complex semi-linked with bordering bone.
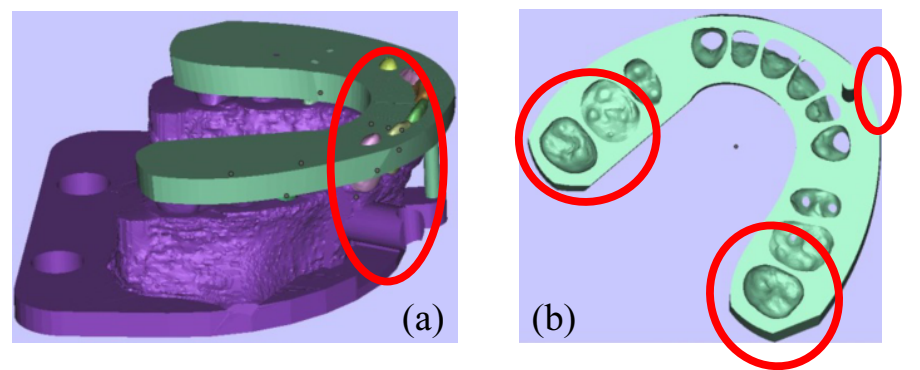

Fig. 4. Guide for teeth insertion (a) guide and oral model (b) guide.
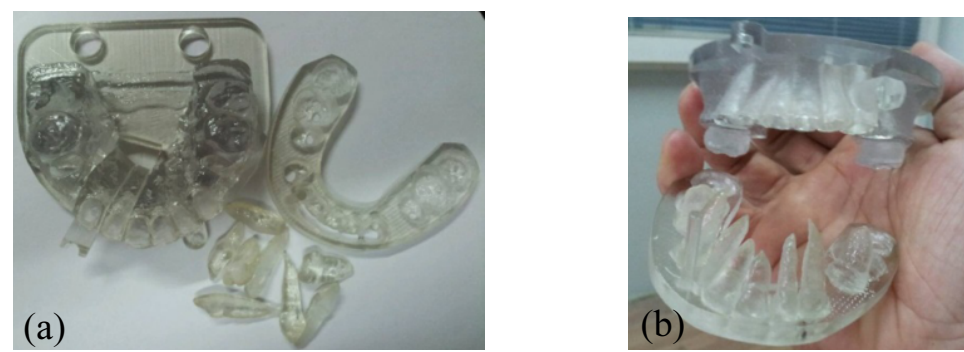

Fig. 5. Fabricated models (a) fabricated models and guide by 3D printing; (b) teeth in guide and maxilla.

\section{Fabrication and assembling}

To get the simulation model, the hard parts in this model including teeth, maxilla and guide should be fabricated separately, and a material with similar mechanical properties to PDL should be found too. 


\section{1. $3 D$ printing}

Different from industrial parts, the oral models were very complex in shape, so conventional manufacturing techniques were not applicable. Additive manufacturing, commonly referred to as 3D printing, which can handle any 3D model represented by triangular mesh, is very appropriate for medical model fabrication [9]. The models of the teeth, maxilla, and guide were fabricated using a 3D printer, Objet Connex 350, a machine from 3D System (Valencia, CA, USA). The models were imported into the control computer of the printer by STL file, and then printed layer by layer with a resin material, FullCure830. The fabricated models are shown in Figure 5.

\subsection{Simulated PDL creation}

In periodontium, bone and tooth are often assumed to be linear elastic during biomechanical analysis, and their mechanical properties, including Young's modulus $(E)$ and Poisson's ratio $(v)$, are reported often. But the PDL, as a key part in this model, show a nonlinear, time-dependent viscoelastic behavior, and have special mechanical properties [10]. However, in actual calculations for biomechanical analysis, the PDL are often simplified to linear elastic for facilitating, and this simplification has been proved to have a limited impact on the results [11]. To acquire a simulated PDL with similar mechanical properties $(E=0.69 \mathrm{MPa}, v=0.4)$, a kind of bi-component silicone was adopted, which consisted of two kinds of gasket sealant (Loctite, Kenkel Corp. Rocky Hill, CT), one of which is a blue hard component, product No. 58730, and the other is a black soft component, product No. 30514. A test model, including a lateral incisor with a measuring bar and the bone socket, was designed and fabricated, as shown in Figure 6(a), and a simplified test system was also constructed with a Nano17 and micrometer, as shown in Figure 6(b). Between the tooth root and socket, a space of $0.25 \mathrm{~mm}$ for the PDL was left, and a line for marking the position of the tooth in the socket was also designed. Three ratios, 1:9, 2:8, and 3:7 of hard component to soft component, were used to simulate the PDL. During testing, the loads applied on the tooth by micrometer by rotating the screw, were measured by Nano17, and the displacement of the tooth was also read by micrometer. Creating the relationship between force and displacement, and comparing it to the preset $E$ and $v$, the ratio of 2:8 was found to be appropriate, as shown in Figure 6(c).

\subsection{Model assembling}

Based on the fabricated parts and PDL material, the model was assembled according to following procedure: (1) the bi-component silicone was filled into each socket of maxilla fully; (2) Each tooth
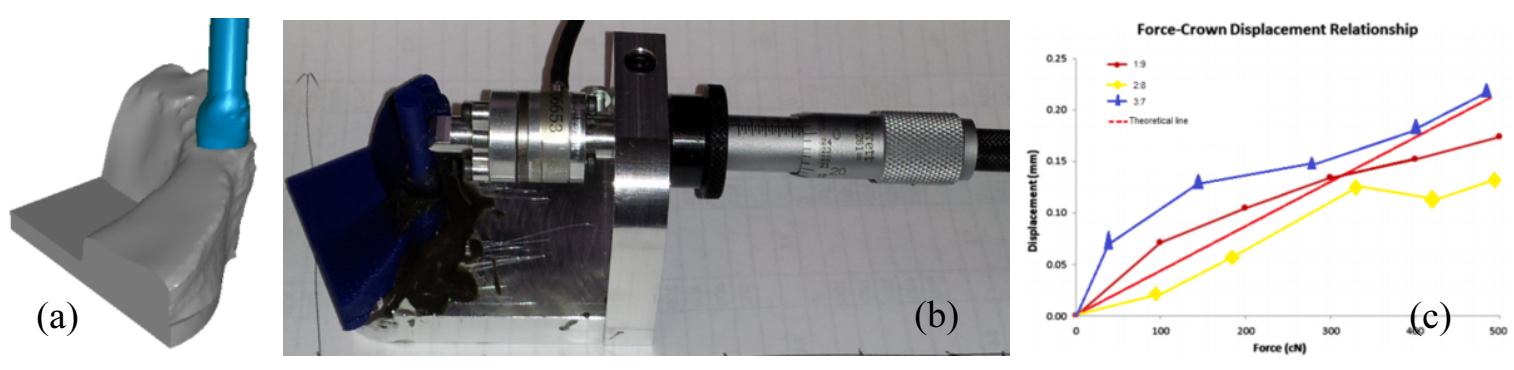

Fig. 6. Experiment on PDL material (a) 3D model for PDL testing (b) testing system (c) Test results. 
was inserted into the middle of the corresponding socket to ensure the tooth root was evenly surrounded by the silicone; (3) The final accurate position of each tooth was placed by the guide and the simulated model was acquired. The model should be kept around 1 hour for silicone stabilization before measuring.

\section{Conclusion}

Based on techniques of digital design and fabrication, an oral model of periodontium was created for biomechanical analysis of orthodontics. To recover the positions of the teeth in the sockets, a guide was used, and with bi-component silicone, PDL with similar mechanical properties were created also, which guaranteed the test data was close to reality with high accuracy.

To realize the 3D force measurements, the test system, including this model, Nano17s, and the frame will be constructed in the future, and more research, including system integration, display software development, and calibration, will be performed as well.

\section{Acknowledgement}

This project is jointly supported by National Natural Science Foundation of China (Grant No. 51375453), Natural Science Foundation of Zhejiang Province (Grant No. LY13E050017), and the Scientific Research Foundation for the Returned Overseas Chinese Scholars, State Education Ministry.

\section{References}

[1] H.M. Badawi, R.W. Toogood, J.P.R. Carey, G. Heo and P.W. Major, Three-dimensional orthodontic force measurements, Am. J. Orthod. Dentofacial Orthop. 136 (2009), 518-528.

[2] J. Chen, W. Li, M.V. Swain, M.A. Darendeliler and Q. Li. A periodontal ligament driven remodeling algorithm for orthodontic tooth movement, J. Biomech. 47 (2014), 1689-1695.

[3] A.V. Schepdael, L. Geris and J.V. Sloten, Analytical determination of stress patterns in the periodontal ligament during orthodontic tooth movement, Medical Engineering \& Physics 35 (2013), 403-410.

[4] J. Schneider, M. Geiger and F.G. Sander, Numerical experiments on long-time orthodontic tooth movement, Am. J. Orthod. Dentofacial Orthop. 121 (2002), 257-265.

[5] C. Canales, M. Larson, D. Grauer, R. Sheats, C. Stevens and C.C. Ko, A novel biomechanical model assessing continuous orthodontic archwire activation, Am. J. Orthod. Dentofacial Orthop. 143 (2013), 281-290.

[6] T.W. Major, J.P. Carey, D.S. Nobes, G. Heo and P.W. Major, Measurement of plastic and elastic deformation due to third-order torque in self-ligated orthodontic brackets, Am. J. Orthod. Dentofacial Orthop. 140 (2011), 326-339.

[7] Y.J. Ren, J.C. Maltha and A.M. Kuijpers-Jagtman, Optimum force magnitude for orthodontic tooth movement: A systematic literature review, The Angle Orthodontist 73 (2003), 86-92.

[8] Catalog of multi-axis force/torque sensor, ATI Industrial automation, 2007, available at http://www.atiia.com/Library/documents/ATI_FT_Catalog.pdf.

[9] M. Martorelli, S. Gerbino, M. Giudice and P. Ausiello, A comparison between customized clear and removable orthodontic appliances manufactured using RP and CNC techniques, Dental Materials 29 (2013), e1-e10.

[10] L.H. Qian, M. Todo, Y. Morita, Y. Matsushita and K. Koyano, Deformation analysis of the periodontium considering the viscoelasticity of the periodontal ligament, Dental materials 25 (2009), 1285-1292.

[11] Z.Y. Xia, F.F. Jiang and J. Chen, Estimation of periodontal ligament's equivalent mechanical parameters for finite element modeling, Am. J. Orthod. Dentofacial Orthop. 143 (2013), 486-49. 